\title{
Leukocyte chemotactic factor 2 amyloidosis (ALECT2) is a common form of renal amyloidosis among Egyptians
}

\author{
Christopher P Larsen ${ }^{1}$, Wesam Ismail ${ }^{2,3}$, Paul J Kurtin ${ }^{4}$, Julie A Vrana ${ }^{4}$, Surendra Dasari ${ }^{4}$ \\ and Samih H Nasr ${ }^{4}$ \\ ${ }^{1}$ Nephropath, Little Rock, AR, USA; ${ }^{2}$ Pathology Department, Beni-Suef University, Beni-Suef, Egypt; ${ }^{3}$ Pathlab, \\ Cairo, Egypt and ${ }^{4}$ Department of Laboratory Medicine and Pathology, Mayo Clinic, Rochester, MN, USA
}

\begin{abstract}
Large case series of renal amyloidosis subtypes have recently been published in the United States and Europe showing AL amyloidosis to be the predominant subtype in this part of the world. However, the most common subtypes of renal amyloidosis throughout the rest of the world are unknown. We present here the first large case series detailing the subtypes of renal amyloidosis among Egyptians. In this population, AA amyloidosis was the most common type of amyloidosis on renal biopsy at $48 \%$. The newly described leukocyte chemotactic factor 2 amyloidosis (ALECT2) was the second most common type and represented nearly one-third of renal amyloid cases at $31 \%$. AL accounted for only $20 \%$ of cases. The pathologic findings in ALECT2 cases were similar to those previously described in other case series. Thus ALECT2, which was initially thought to affect mainly Hispanics in the United States, appears to represent an important and likely underrecognized etiology of chronic kidney disease among Egyptians and probably in other ethnic groups around the world.
\end{abstract}

Modern Pathology (2016) 29, 416-420; doi:10.1038/modpathol.2016.29; published online 12 February 2016

Amyloidosis is the abnormal accumulation of misfolded protein in the parenchyma of organs, often resulting in organ failure. There are now more than 30 different types of amyloidosis described in humans. ${ }^{1}$ Leukocyte chemotactic factor 2 amyloidosis (ALECT2) is one of the most recently described forms, which most commonly affects the kidney and liver. ${ }^{2}$ Currently there are no known biomarkers for this disease and the only way to diagnose it is by biopsy. Therefore, the prevalence of this disease is unknown and is likely underrecognized due to the fact that slowly progressive renal failure with bland urine sediment, the most common clinical presentation in ALECT2, is not an indication for renal biopsy.

ALECT2 was described in the United States where it has been shown to primarily affect Hispanic patients of Mexican descent. ${ }^{3,4}$ The worldwide distribution of this disease is not currently known though case reports of disease in patients of various ethnicities are emerging. These include First Nations People from Canada, ${ }^{5}$ Native Americans, ${ }^{6,7}$

Correspondence: Dr CP Larsen, MD, Nephropath, 10810 Executive Center Drive, Suite 100, Little Rock, AR 72211, USA.

E-mail: chris.larsen@nephropath.com

Received 9 October 2015; revised 24 December 2015; accepted 3 January 2016; published online 12 February 2016
Punjabi, ${ }^{8,9}$ Sudanese, ${ }^{10}$ and Chinese. ${ }^{11}$ In the United States, there is a relatively low disease burden in Caucasians and African Americans. In this study, we have attempted to further characterize the burden of ALECT2 in the Egyptian population by typing renal amyloid cases from a large biopsy center in this country.

\section{Materials and methods}

The database from Pathlab in Cairo, Egypt was searched for cases of amyloidosis between January 2012-May 2015. A total of 161 formalin fixed paraffin embedded kidney biopsies with amyloidosis were identified during this time period. All cases were processed at Pathlab by light and immunofluorescence microscopy using routine techniques, previously described. ${ }^{12}$ Briefly, kidney biopsies were fixed in buffered formalin, dehydrated in graded alcohols, and embedded in paraffin using standard techniques. Serial $3 \mu \mathrm{m}$-thick sections were cut and treated with hematoxylin and eosin, Jones methenamine silver, Masson trichrome, and periodic acid-Schiff reagent. The diagnosis of amyloidosis was made based on Congo red staining.

These blocks were sent to Nephropath and Mayo Clinic for typing of the amyloidosis. A total of 116 

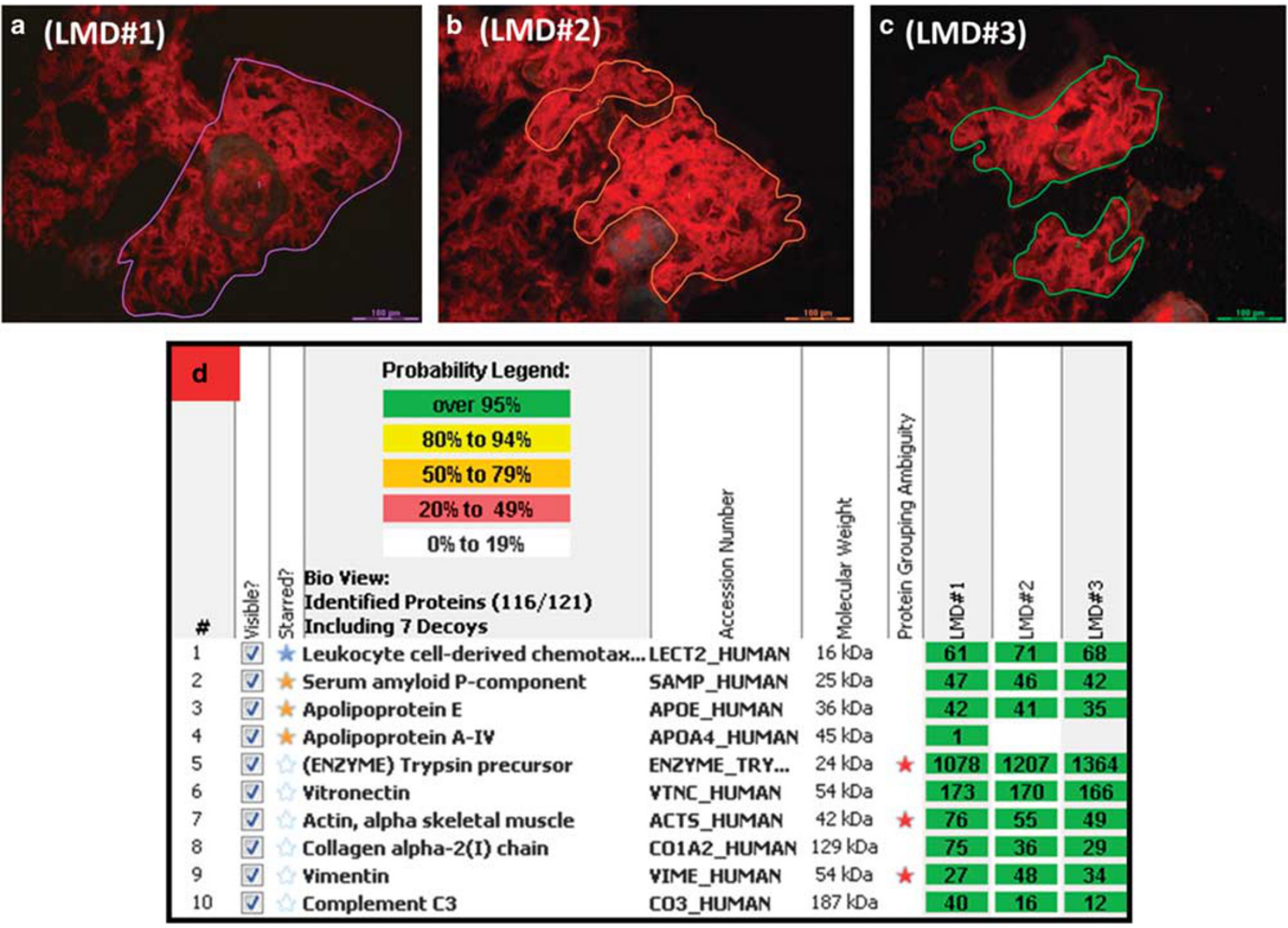

Figure 1 Laser microdissection/mass spectrometry in a patient with renal ALECT2 amyloidosis. (a-c) Renal parenchymal congophilic amyloid deposits from a patient with ALECT2 are visualized under fluorescent light and marked for microdissection. Three separate microdissections (LMD\#1, LMD\#2, and LMD\#3) are analyzed. (d) Scaffold software display of amyloid-associated proteins identified within the deposits by liquid chromatography/tandem mass spectrometry. The three columns on the right represent the separate micro-dissected samples run in triplicate. The numbers within the columns represent the total number of mass spectra corresponding to the identified protein and the color of the box reflects the probability that the spectra are correctly assigned to the identified protein (all $>95 \%$ ). In this case, the presence of abundant spectra for serum amyloid $\mathrm{P}$ and apolipoprotein $\mathrm{E}$ is indicative of amyloid, whereas the presence of abundant spectra for leukocyte cell-derived chemotaxin 2 (LECT2) establishes the identity of the amyloid as ALECT2.

blocks had sufficient tissue for amyloid typing. A Congo red stain was performed in all cases in addition to serial sections for staining. Immunoperoxidase stains for AA (Dako, Carpenteria, CA, USA) and LECT2 (R\&D Systems, Minneapolis, $\mathrm{MN}$, USA) as well as paraffin immunofluorescence for $\kappa$ and $\lambda$ (both from Dako, Carpenteria, CA, USA) were performed in all cases. Detailed methods for the AA and LECT2 staining procedures are provided in Supplementary Tables 1 and 2, respectively. Cases that were not able to be typed using these stains were sent to Mayo Clinic for typing of the amyloidosis by mass spectroscopy as previously described (Figure 1). ${ }^{13}$ This included cases with negative or weak staining in the amyloid deposits by immunohistochemistry.

The extent of amyloid deposition in different compartments of the kidney was evaluated by reviewing the Congo red stained slides. Glomerular amyloid deposits were scored with a semi-quantitative scale as follows: 0, absent; 1, amyloid deposits affecting $\sim 25 \%$ of the glomerulus; $2,25-50 \%$ of the glomerulus, and $3,>50 \%$ of the glomerulus. The extent of amyloid deposition in the cortical and medullary interstitium was scored as: 0 , absent; 1 , $25 \%$ of parenchyma involved; $2,25-50 \%$, and 3 , $>50 \%$. The extent of amyloid deposition in arteries was scored as: 0 , absent; 1 ; mild, 2; moderate; and 3, severe.

\section{Results}

The results of the amyloid type in this case series are shown in Table 1. ALECT2 was the second most common form of amyloidosis in this population behind AA. ALECT2 primarily presented in patients with a mean age of 59.1. There were 20 males and 16 females with ALECT2. By comparison, AA patients had a mean age of 44.9 years and AL patients had a 
Table 1 Amyloid type in a series of cases from Egypt

\begin{tabular}{lccc}
\hline Amyloid type & Number (\%) & Mean age (range) & Male/female \\
\hline AA & $56(48 \%)$ & $44.9(13-77)$ & $32 / 24$ \\
ALECT2 & $36(31 \%)$ & $59.1(30-83)$ & $20 / 16$ \\
AL & $23(20 \%)$ & $56.1(24-70)$ & $13 / 10$ \\
AFib & $1(1 \%)$ & 20 & $1 / 0$ \\
Total & 116 & $51.3(13-83)$ & $66 / 50$ \\
\hline
\end{tabular}

mean age of 56.1 years. The etiology of the AA amyloid was unknown in the majority of cases though, among the 56 cases, there were 9 cases with familial mediterranean fever and 2 cases associated with rheumatoid arthritis.

The pathologic distribution of ALECT2 is shown in Table 2 and Figure 2. The amyloid deposits were present in the renal cortical interstitium in all cases. Most cases showed evidence of glomerular and vascular deposition as well. The medullary interstitium was the compartment with the least involvement and, when present, the deposits were often sparse. The amyloid deposits showed bright red congophilia in all cases with green birefringence upon polarization. Among the ALECT2 cases there were $26(72 \%)$ with no other significant histologic findings on biopsy. Ten of the cases had additional histologic diagnoses including five cases with arterionephrosclerosis (14\%), two cases with diabetic glomerulosclerosis $(6 \%)$, and one case each of membranous glomerulopathy (3\%), acute tubular injury (3\%) and interstitial nephritis (3\%). Most cases were typed by immunohistochemical staining without mass spectroscopy as there were no cases with more than one antibody positive in the amyloid deposits. Mass spectroscopy was utilized for amyloid typing in 14 cases due to either weak positive staining in the amyloid deposits by the LECT2 stain (nine cases) or no staining by any of the antibodies (five cases). All nine cases with weak LECT2 staining had an amyloid type that matched by mass spectroscopy. The five cases with negative staining included two cases of ALECT2, one case of fibrinogen A $\alpha$-chain amyloidosis, and 2 cases of $\mathrm{AL}$ $\lambda$ amyloidosis.

\section{Discussion}

We present the first large case series of renal amyloidosis subtypes in Egyptian patients. ALECT2 was found to be significantly more common in this population than has been described in other case series of renal amyloidosis. The largest renal amyloid case series reported in the United States (474 cases) showed AL to be the most common amyloid subtype identified, representing $85.9 \%$ of amyloidosis cases followed by AA at $7 \%$ and ALECT2 at $2.7 \% .{ }^{14} \mathrm{~A}$ second large renal amyloid study in the US (414 total cases) showed similar findings with AL representing $83.3 \%$ of amyloid cases. However, focusing on the
Table 2 Location of ALECT2 deposits on renal biopsy

\begin{tabular}{lcc}
\hline & $\begin{array}{c}\text { Number of cases } \\
\text { with compartment } \\
\text { affected (\%) }\end{array}$ & $\begin{array}{c}\text { Mean extent of } \\
\text { deposition (0-3) }\end{array}$ \\
\hline Glomerular & $27 / 32(84)$ & 2.0 \\
Arteries & $25 / 33(76)$ & 1.6 \\
Cortex interstitium & $36 / 36(100)$ & 2.9 \\
Medulla interstitium & $10 / 18(56)$ & 0.7 \\
\hline
\end{tabular}

amyloidosis type in renal biopsies that came from the Southwest US, a very different picture emerged with $54 \%$ of cases showing ALECT2 and $41 \%$ AL. This is likely due to the fact that there is a much higher population of patients with Mexican ethnicity in this region of the country. Renal amyloid case series from Germany and Switzerland have also shown AL to be the most common type of amyloidosis at $53.2 \%$ but, similar to the Egyptian data, had a much higher burden of AA at $40.3 \%{ }^{15}$ No cases of ALECT2 were reported in these European series; however, it was not specifically tested for. By comparison, our current Egyptian series shows AA amyloidosis to be the most common at $48 \%$ with ALECT2 representing the second most common at $31 \%$ followed by AL at $20 \%$. Clearly the prevalence of the amyloidosis subtypes varies widely in different ethnic groups. It is likely that other populations with a high disease burden of ALECT2 will emerge as awareness of the disease increases.

The clinicopathologic findings in Egyptian ALECT2 patients are similar to those previously described in the literature. The disease was most often seen in elderly patients without gender preference. The Egyptian cohort was slightly younger at 59 years than the previously described US cohorts, which were primarily comprised of Hispanics and had a mean age of 69 years. $^{2}$ This Egyptian cohort also includes the youngest person reported with ALECT2 in the kidney to date at the age of 30 years. AA and AL patients in the Egyptian cohort were also younger than the previously described US cohorts. ${ }^{14}$ This could be in part due to a shorter life expectancy in Egypt compared with the US (71 vs 79 years) resulting in underrepresentation of elderly patients with amyloidosis in the Egyptian cohort. ${ }^{16}$ The morphologic pattern of deposition in the kidney was similar to that previously described in other large case series with amyloid predominantly affecting the cortical interstitium (Table 2). The precise etiology of ALECT2 remains unknown. The strong predilection for certain ethnic groups would suggest a genetic component but, thus far, no disease causing variants have been identified. ${ }^{7}$ It is hypothesized that overproduction of ALECT2 by the liver leads to deposition in the parenchyma of various organs. ${ }^{2}$ All ALECT2 patients with sequencing of the LECT2 gene have been found to be homozygous for the 

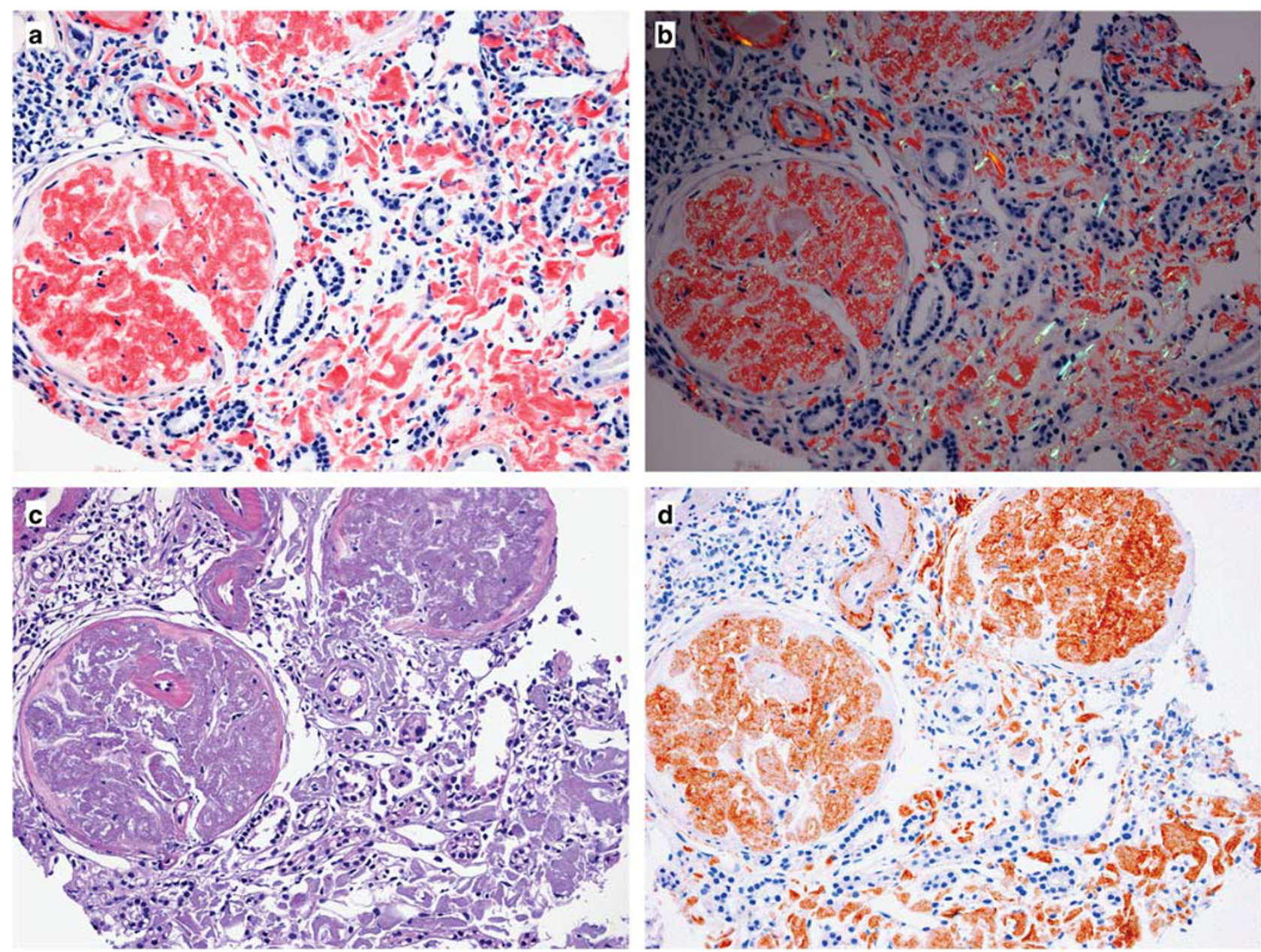

Figure 2 Leukocyte chemotactic factor 2 amyloidosis. (a and b) Uptake of Congo red by glomerular and interstitial amyloid deposits (a) with green birefringence upon (b) polarization (original magnification $\times 200$ ). (c) Extensive amorphous deposits of LECT2 amyloid in the glomeruli and interstitial compartments (hematoxylin and eosin; original magnification $\times 200$ ). (d) Reactivity of glomerular and interstitial amyloid deposits with an anti-LECT2 antibody (immunoperoxidase technique, $\times 200$ ).

G nucleotide in a non-synonymous SNP at position 172 (SNP rs31517). This polymorphism is common with an allele frequency of 0.51 in patients of Mexican ancestry. The overrepresentation of this common polymorphism in patients with ALECT2 indicates that it is necessary for disease to develop but not the sole etiology of the disease. It has been hypothesized that this SNP increases the amyloidogenic propensity of the protein. ${ }^{17}$ It is also possible that a gene environment interaction results in disease. Future studies directed at determining the underlying etiology of ALECT2 are desperately needed.

An invasive procedure such as renal biopsy is the only way to make the diagnosis of ALECT2. Deposition has previously been reported in the multiple organs including the spleen, liver, prostate, and pancreas. ${ }^{6}$ However, there have been no reports of deposition in the skin or fibroadipose tissue to suggest that a less invasive diagnostic procedure such as fat pad aspirate or skin biopsy would be useful. ALECT2 most commonly presents with slowly progressive renal failure and we have shown that they often have comorbid illnesses such as diabetes mellitus and hypertension. Approximately $40 \%$ of the patients do not have significant proteinuria. $^{7}$ Since slowly progressive renal failure with bland urine sediment is not typically an indication for renal biopsy, particularly when the patient has comorbid illnesses that are known to lead to renal disease, we believe ALECT2 is likely an underrecognized etiology of chronic kidney disease in certain ethnicities. Currently, there is no specific treatment known to be efficacious for the treatment of ALECT2. However, it is important to identify this type of amyloidosis so to prevent mistreatment of the disease with the chemotherapeutic agents that are commonly used for treatment of AL amyloidosis. Most patients have deterioration of renal function after diagnosis with one study showing a mean drop in eGFR of $0.5 \mathrm{ml} / \mathrm{min} / 1.73 \mathrm{~m}^{2}$ per month. ${ }^{7}$ Another study showed independent predictors of renal survival were serum creatinine at diagnosis (with a value of $2.0 \mathrm{mg} / \mathrm{dl}$ being the best cutoff for predicting ESRD), 
percentage global glomerulosclerosis, and presence of diabetes. ${ }^{6}$

We present the first large case series detailing the subtypes of renal amyloidosis among Egyptians and show that this population has a unique profile of amyloidosis compared with other large renal amyloid case series. Unlike case series from the Europe and the United States, where AL predominates, AA is the most common type of amyloid identified among Egyptians. Even more surprising is that the newly described LECT2 amyloidosis is the second most common and represents nearly one-third of renal amyloid cases in this population. This emerging disease appears to represent an important and likely underrecognized etiology of chronic kidney disease in various ethnic groups around the world.

\section{Disclosure/conflict of interest}

The authors declare no conflict of interest.

\section{References}

1 Sipe JD, Benson MD, Buxbaum JN et al. Nomenclature 2014: Amyloid fibril proteins and clinical classification of the amyloidosis. Amyloid 2014;21:221-224.

2 Nasr SH, Dogan A, Larsen CP. Leukocyte cell-derived chemotaxin 2-associated amyloidosis: A recently recognized disease with distinct clinicopathologic characteristics. Clin J Am Soc Nephrol 2015;10:2084-2093.

3 Benson MD, James S, Scott K et al. Leukocyte chemotactic factor 2: a novel renal amyloid protein. Kidney Int 2008;74:218-222.

4 Larsen CP, Walker PD, Weiss DT et al. Prevalence and morphology of leukocyte chemotactic factor 2-associated amyloid in renal biopsies. Kidney Int 2010;77:816-819.

5 Hutton HL, DeMarco ML, Magil AB et al. Renal leukocyte chemotactic factor 2 (LECT2) amyloidosis in first nations people in northern British Columbia, Canada: a report of 4 cases. Am J Kidney Dis 2014;64: 790-792.

6 Said SM, Sethi S, Valerie AM et al. Characterization and outcomes of renal leukocyte chemotactic factor 2associated amyloidosis. Kidney Int 2014;86:370-377.

7 Larsen CP, Kossmann RJ, Beggs ML et al. Clinical, morphologic, and genetic features of renal leukocyte chemotactic factor 2 amyloidosis. Kidney Int 2014;86: 378-382.

8 Dogan A, Theis JD, Vrana JA. Clinical and pathological phenotype of leukocyte cell-derived chemotaxin-2 (LECT2) amyloidosis (ALECT2). Amyloid 2010;17: 69-70.

9 Kulkarni U, Valson A, Korula A et al. Leukocyte derived chemotaxin 2 (ALECT2) Amyloidosis. Mediterr J Hematol Infect Dis 2015;7:e2015043.

10 Holanda DG, Acharya VK, Dogan A et al. Atypical presentation of atypical amyloid. Nephrol Dial Transplant 2011;26:373-376.

11 Wang Y, Wang S, Zhang Y. Leukocyte chemotactic factor 2 associated renal amyloidosis: one case report. J Peking Univ 2015;47:349-351.

12 Walker PD, Cavallo T, Bonsib SM. Practice guidelines for the renal biopsy. Mod Pathol 2004;17: 1555-1563.

13 Vrana JA, Gamez JD, Madden BJ et al. Classification of amyloidosis by laser microdissection and mass spectrometry-based proteomic analysis in clinical biopsy specimens. Blood 2009;114:4957-4959.

14 Said SM, Sethi S, Valeri AM et al. Renal amyloidosis: origin and clinicopathologic correlations of 474 recent cases. Clin J Am Soc Nephrol 2013;8: 1515-1523.

15 von Hutten $\mathrm{H}$, Mihatsch M, Lobeck H et al. Prevalence and origin of amyloid in kidney biopsies. Am J Surg Pathol 2009;33:1198-1205.

16 Life expectancy at birth, total (years). World Development Indicators 2015. Available at http://data.worldbank.org/indicator/SP.DYN.LE00.IN.

17 Murphy CL, Wang S, Kestler D et al. Leukocyte chemotactic factor 2 (LECT2)-associated renal amyloidosis: a case series. Am J Kidney Dis 2010;56: 1100-1107.

$($ (1) $\odot \odot$ This work is licensed under a Creative Commons Attribution-NonCommercialNoDerivs 4.0 International License. The images or other third party material in this article are included in the article's Creative Commons license, unless indicated otherwise in the credit line; if the material is not included under the Creative Commons license, users will need to obtain permission from the license holder to reproduce the material. To view a copy of this license, visit http://creativecommons.org/licenses/ by-nc-nd/4.0/

Supplementary Information accompanies the paper on Modern Pathology website (http://www.nature.com/ modpathol) 